\title{
Mitochondria disease due to humidifier disinfectants: diagnostic criteria and its evidences
}

\author{
Jong Han Leem ${ }^{1 *}(\mathbb{D}$, Hwan-Cheol Kim*i(D) \\ Department of Occupational and Environmental Medicine, Inha University, Incheon, Korea
}

\begin{abstract}
Humidifier disinfectant damages caused by the misuse of humidifier disinfects, such as polyhexamethylene guanidine (PHMG), resulted in chemical disasters in South Korea in 2011. About four million people were exposed to humidifier disinfectants (HDs) in the 17 years between 1994 and 2011. Although fatal lung damage was initially reported, investigations into the victims' injuries revealed that the damage was not limited to the lungs, but that systemic damage was also confirmed. Considering the spread of HD from the lungs to the whole body, the toxic effects of PHMG from reactive oxygen species (ROS), NOTCH signaling pathways, and mitochondrial dysfunction resulted in endothelial damage in the lungs, blood vessels, liver, kidneys, bone marrow, nerves, and muscles. The main toxic mechanisms involved in HD damage may be the NOTCH pathway and mitochondrial damage. There are many case reports which include neurologic disorders (ADHD, depression, posttraumatic stress disorder), muscular disorder (exercise intolerance, myalgia), energy metabolism disorder (chronic fatigue syndrome), and immunologic disorder (rheumatoid arthritis) in HDs victims. These case reports involve multi-system involvement in HDs victims. Further well-designed study is needed to clarify whether mitochondrial dysfunction is associated with multi-organs involvement in HDs victims.
\end{abstract}

Keywords: Humidifier disinfectants, PHMG, mitochondria diseases, reactive oxygen species, NOTCH pathway, Humidifier disinfectants syndrome

\section{Introduction: Humidifier disinfectants (HDs) study results so far achieved}

From 2002 through 2020, about 1,600 people were reported to die of fatal injuries associated with the use of humidifier disinfectants (HDs) in South Korea. Several chemical disinfectants used for household humidifiers were later clinically confirmed to cause HD-associated lung injury (HDLI). Polyhexamethylene guanidine phosphate (PHMG-P) is the main ingredient of the HDs associated with lung disease. Epidemiological studies, animal studies, and dose-response analysis demonstrated a strong association between HDs use and lung injuries. The diagnostic criteria for HDLI was defined on the basis of the clinical, pathological, and radiological attributes of the patients [1]. Because of the fatal lung injuries in South Korea, investigations into the adverse health effects of HDs have

Received: April 16, 2020 Accepted: May 13, 2020

Corresponding author: Jong Han Leem

Department of Occupational and Environmental Medicine, Inha University College of Medicine, 27 Inhang-ro, Jung-gu, Incheon 22332, Korea

E-mail: ekeeper21@naver.com

This article is available from: https://eaht.org/ focused on the lung toxicity of PHMG-P [2]. Therefore, the following toxicologic studies focused on the association between HDs and pulmonary fibrosis. PHMG-P aerosol particles induce pulmonary inflammatory and fibrotic responses [3]. As PHMG-P-induced lung fibrosis is different from that induced by known fibrogenic agents, such as bleomycin, it is important to understand the molecular mechanisms underlying this effect. Guanidine-based disinfectants, PHMG-P, polyhexamethylene biguanide (PHMB), and oligo (2- (2-ethoxy) ethoxyethyl guanidinium chloride (PGH) induce epithelial-mesenchymal transition in A549 alveolar epithelial cells [4]. PHMG-P promotes reactive oxygen species (ROS) generation and consequently increases the expression of DNA damage markers, such as ATM and H2AX phosphorylation [5]. NOTCH1 transcripts significantly increased in lung tissues from HDLI cases compared to unexposed controls ( $\mathrm{p}=0.05)$, so NOTCH1 may play an important role in the pulmonary fibrosis of HDLI [6]. PHMG-P induced epithelial-mesenchymal transition (EMT) through Akt/NOTCH signaling pathways and ZEB2 played an important role in PHMG-P-induced lung toxicity [7].

Age-period-cohort (APC) analysis using health insurance data showed that many diseases, such as asthma, bronchiec- 
tasis, interstitial lung disease (ILD), pneumonia, and toxic hepatitis, are associated with exposure to HDs [8]. According to a recent survey [9], the high prevalence of symptoms or chronic diseases, such as ophthalmologic diseases, nasal diseases, cardiovascular disease, chronic fatigue, attention-deficit/hyperactivity disorder (ADHD), and developmental disorders, may be related to HDs. Are there common underlying toxic mechanisms for all these diseases, including HDLI? NOTCH signaling on mitochondrial proteome, which in turn affects the functioning of key metabolic pathways, connects an important signaling pathway to the regulation of cellular metabolism [10]. The NOTCH-activated signaling cascade interacts with mitochondrial remodeling proteins to regulate cell survival [11]. A previous study reported that HDLI is caused by the NOTCH-activated signaling cascade. Interestingly, interstitial fibrosis can be caused by the NOTCH-activated signaling cascade. Many researchers have identified and described the underlying processes that result in metabolic dysregulation, metabolic reprogramming, and mitochondrial dysfunction observed in the cells of idiopathic pulmonary fibrosis (IPF) lungs [12]. Mitochondrial dysfunction is the molecular treatment target of IPF [13]. To identify the mechanism of PHMG hepatotoxicity, HepG2 cells were exposed to PHMG$\mathrm{P}$ for $72 \mathrm{hr}$. The cell viability was significantly decreased by PHMG-P in time- and concentration-dependent manners. The mitochondrial membrane potential was markedly reduced by PHMG-P and the apoptotic signaling cascade was activated [14].

\section{New hypothesis about mitochondrial diseases}

Many studies already showed that biocides can cause various non-communicable diseases (NCD) by inducing mitochondria dysfunction. Typical examples are organophosphate, herbicide, and pyrethroids [15-17].

PHMG overexposure leads to metabolic overload of the mitochondria and the damaged mitochondria are likely to result in muscle insulin resistance and $\beta$-cell dysfunction [18]. Unexplained cardiovascular disease, such as angina pectoris and myocardial infarction at young ages, could be associated with exposure to PHMG. Cardiovascular toxicity caused by PHMG was observed in an animal study [19]. Mitochondria in airway epithelium, smooth muscle, and fibroblasts play differential roles, being consistent with their contributions to disease biology, such as asthma and chronic obstructive pulmonary disease (COPD) [20]. Mitochondrial dysfunction could trigger developing asthma and COPD. ROS and mitochondrial dysfunction could be the cause of bronchiectasis. Mitochondria dysfunction could play a core role in developing immune disorders such as rheumatoid arthritis [21].

Mitochondrial defects were detected in ADHD cybrids created by patients' platelets, implying that a bioenergetic crisis in the mitochondria could be a contributory factor in ADHD pathology and/or phenotypes [22]. Mitochondrial dysfunction could play a critical role in cancer progression, cognitive decline, and repeated stroke at young ages [23-25].

Toxicological studies to date have tried to show an association between HDs exposure and HDLI. Researchers have tried to elucidate the mechanism of pulmonary fibrosis but did not investigate the toxicological mechanisms of asthma, interstitial pneumonia, pneumonia, toxic hepatitis, cancer, chronic fatigue syndrome or ADHD.

Considering the movement of HD in the lungs, which spreads from the lungs to the whole body [26], the toxic effects of PHMG on pulmonary epithelial cells through ROS and the NOTCH signaling pathways, and mitochondrial dysfunction have also been found in blood vessels, liver, kidneys, bone marrow, nerves, and muscles. Bone marrow damage can be associated with idiopathic pulmonary fibrosis by abnormal immune modulation of immune cells [27].

HDs were shown to exert typical toxicologic effects on various target organs, such as the skin, conjunctiva, nasal mucosa, bronchial mucosa, and alveoli, which shared common toxicological responses. Diverse diseases can occur at the same time with a common toxicologic mechanism and cause humidifier disinfectant syndrome (HDS) [28].

Mitochondria disease criteria was suggested by E. Morava et al. [29] Mitochondria diseases due to HDs can be diagnosed as follows. First, exposure to HDs should be confirmed. Second, upper or lower respiratory tract diseases, such as asthma, interstitial lung disease, bronchiectasis, pneumonia, and COPD should be confirmed. Third, mitochondria diseases criteria should be met. Fourth, Other causes, such as genetic factor, radiation exposure, and other occupational and environmental exposures should be ruled out.

HD victims are at increased risk of developing many HD-related diseases. However, HD exposure no longer occurs because the use of HD was banned in 2011. Thus, why do HD-related diseases develop and continue? Damaged lymphocyte are still observed in many HDs victims. This can be due to HDinduced genetic mutations [30] and epigenetic changes [31] in the mitochondria.

Mitochondrial diseases are very important because most NCDs are associated with these diseases. So, to investigate into the possible environmental causes of mitochondrial diseases has tremendous importance. To clarify the association 
between HDs and mitochondrial disease can be good step to prevent NCDs.

To treat and prevent these kinds of diseases, the concept of HDS should be considered. Many therapies to target mitochondrial dysfunction are being developed [32,33] and data has accumulated on the toxicologic mechanism of HDs. Therefore, it is necessary to update the diagnostic criteria for HD-related diseases and establish strategies to treat and prevent HD-related diseases to care for HDs victims.

\section{Conclusion}

There are many case reports which include neurologic disorders (ADHD, depression, posttraumatic stress disorder), muscular disorder (exercise intolerance, myalgia), energy metabolism disorder (chronic fatigue syndrome), and immunologic disorder (rheumatoid arthritis) in HDs victims. These case reports involve multi-system involvement in HDs victims. Further well-designed study is needed to clarify whether mitochondrial dysfunction is associated with multi-organs involvement in HD victims.

\section{Declaration of Competing Interest}

The authors declare that they have no known competing financial interests or personal relationships that could have appeared to influence the work reported in this paper.

\section{CRediT author statement}

JHL: Conceptualization; Writing - Original Draft; HCK: Writing - Reviewing \& Editing

\section{References}

1. Kim WY, Hong SB. Humidifier disinfectant-associated lung injury: six years after the tragic event. Tuberc Respir Dis 2017;80(4):351-357.

2. Kim HR, Hwang GW, Naganuma A, Chung KH. Adverse health effects of humidifier disinfectants in korea: lung toxicity of polyhexamethylene guanidine phosphate. J Toxicol Sci 2016;41(6):711-717.

3. Kim HR, Lee K, Park CW, Song JA, Shin DY, Park YJ, et al. Polyhexamethyleneguanidine phosphate aerosol particles induce pulmonary inflammatory and fibrotic responses. Arch Toxicol. 2016;90(3):617632.

4. Park YJ, Jeong MH, Bang IJ, Kim HR, Chung KH. Guanidine-based disinfectants, polyhexamethylene guanidine-phosphate (PHMG-P), polyhexamethylene biguanide(PHMB), and oligo(2-(2-ethoxy)ethoxyethyl guanidinium chloride (PGH) induced epithelial-mesenchymal transition in A549 alveolar epithelial cells. Inhal Toxicol. 2019; 31(4):161-166.
5. Park JS, Park YJ, Kim HR, Chung KH. Polyhexamethylene guanidine phosphate-induced ROS-mediated DNA damage caused cell cycle arrest and apoptosis in lung epithelial cells. J Toxicol Sci. 2019;44(6): 415-424.

6. Lee E, Kang MJ, Kim JH, Lee SH, Lee SY, Cho HJ, et al. NOTCH1 pathway is involved in polyhexamethylene guanidine-induced humidifier disinfectant lung injuries. Yonsei Med J. 2020;61(2):186-191.

7. Jeong MH, Kim HR, Park YJ, Chung KH. Akt and notch pathways mediate polyhexamethylene guanidine phosphate-induced epithelialmesenchymal transition via ZEB2. Toxicol Appl Pharmacol. 2019;380: 114691.

8. Korean Society of Environmental Health and Toxicology (KOSEHT). Study for improving recognition and judgement standard of health damage of humidifier disinfectant (II). 2019.

9. Korean Society of Epidemiology. Household survey for the victims due to humidifier disinfectants. 2020.

10. Basak NP, Roy A, Banerjee S. Alteration of mitochondrial proteome due to activation of Notch1 signaling pathway. J Biol Chem. 2014; 289(11):7320-7334.

11. Perumalsamy LR, Nagala M, Sarin A. Notch-activated signaling cascade interacts with mitochondrial remodeling proteins to regulate cell survival. Proc Natl Acad Sci USA. 2010;107(15):6882-6887.

12. Zank DC, Bueno M, Mora AL, Rojas M. Idiopathic pulmonary fibrosis: aging, mitochondrial dysfunction, and cellular bioenergetics. Front Med (Lausanne). 2018;5:10.

13. Mora AL, Rojas M, Pardo A, Selman M. Emerging therapies for idiopathic pulmonary fibrosis, a progressive age-related disease. Nat Rev Drug Discov. 2017;16(11):810.

14. Kim SH, Kwon D, Lee S, Ki SH, Jeong HG, Hong JT, et al. . Polyhexamethyleneguanidine phosphate-induced cytotoxicity in liver cells is alleviated by Tauroursodeoxycholic Acid (TUDCA) via a reduction in endoplasmic reticulum stress. Cells. 2019;8(9):1023.

15. Singh N, Lawana V, Luo J, Phong P, Abdalla A, Palanisamy B, et al. Organophosphate pesticide chlorpyrifos impairs STAT1 signaling to induce dopaminergic neurotoxicity: Implications for mitochondria mediated oxidative stress signaling events. Neurobiol Dis. 2018;117:82113.

16. Huang CL, Chao CC, Lee YC, Lu MK, Cheng JJ, Yang YC, et al. Paraquat induces cell death through impairing mitochondrial membrane permeability. Mol Neurobiol. 2016;53(4):2169-2188.

17. Agrawal S, Singh A, Tripathi P, Mishra M, Singh PK, Singh MP. Cypermethrin-induced nigrostriatal dopaminergic neurodegeneration alters the mitochondrial function: a proteomics study. Mol Neurobiol. 2015;51(2):448-465.

18. Sarparanta J, García-Macia M, Singh R. Autophagy and mitochondria in obesity and type 2 diabetes. Curr Diabetes Rev. 2017;13(4):352-369.

19. Kim JY, Kim HH, Cho KH. Acute cardiovascular toxicity of sterilizers, PHMG, and PGH: severe inflammation in human cells and heart failure in zebrafish. Cardiovasc Toxicol. 2013;13(2):148-160.

20. Prakash YS, Pabelick CM, Sieck GC. Mitochondrial dysfunction in airway disease. Chest. 2017;152(3):618-626.

21. Fearon U, Canavan M, Biniecka M, Veale DJ. Hypoxia, mitochondrial dysfunction and synovial invasiveness in rheumatoid arthritis. Nat Rev Rheumatol. 2016;12(7):385-397.

22. Verma P, Singh A, Nthenge-Ngumbau DN, Rajamma U, Sinha S, Mukhopadhyay K, et al. Attention deficit-hyperactivity disorder suffers 
from mitochondrial dysfunction. BBA Clin. 2016;6:153-158.

23. Hsu CC, Tseng LM, Lee HC. Role of mitochondrial dysfunction in cancer progression. Exp Biol Med (Maywood). 2016;241(12):12811295.

24. Grimm A, Friedland K, Eckert A. Mitochondrial dysfunction: the missing link between aging and sporadic Alzheimer's disease. Biogerontol. 2016;17(2):281-296.

25. He Z, Ning N, Zhou Q, Khoshnam SE, Farzaneh M. Mitochondria as a therapeutic target for ischemic stroke. Free Radic Biol Med. 2020;146: 45-58.

26. Shim HE, Lee JY, Lee CH, Mushtaq S, Song HY, Song L, et al. Quantification of inhaled aerosol particles composed of toxic household disinfectant using radioanalytical method. Chemosphere. 2018;207:649654.

27. Cárdenes N, Álvarez D, Sellarés J, Peng Y, Corey C, Wecht S, et al. Senescence of bone marrow-derived mesenchymal stem cells from patients with idiopathic pulmonary fibrosis. Stem Cell Res Ther. 2018
Sep 26;9(1):257.

28. Leem JH, Lee JH. Humidifier disinfectant-associated specific diseases should be called together as "humidifier disinfectant syndrome". Environ Health Toxicol. 2017;32.

29. Morava E, van den Heuvel L, Hol F, de Vries MC, Hogeveen M, Rodenburg RJ, et al. Mitochondrial disease criteria: diagnostic applications in children. Neurol. 2006;67(10):1823-1826.

30. Alston CL, Rocha MC, Lax NZ, Turnbull DM, Taylor RW. The genetics and pathology of mitochondrial disease. J Pathol. 2017;241(2):236250.

31. Sharma N, Pasala MS, Prakash A. Mitochondrial DNA: Epigenetics and environment. Environ Mol Mutagen. 2019;60(8):668-682.

32. El-Hattab AW, Zarante AM, Almannai M, Scaglia F. Therapies for mitochondrial diseases and current clinical trials. Mol Genet Metab. 2017;122(3):1-9.

33. Zhang L, Zhang Z, Khan A, Zheng H, Yuan C, Jiang H. Advances in drug therapy for mitochondrial diseases. Ann Transl Med. 2020;8(1):17. 